\title{
THE ROLE OF CIRCADIAN RHYTHMS AMONG MEDICAL STUDENTS IN TIME MANAGEMENT ORGANIZATION AND ACADEMIC ACHIEVEMENT
}

\author{
Yuliya Modna, ${ }^{1}$ Bernadette Scott $^{2}$
}

\begin{abstract}
Time management is one of the biggest problems of medical students. In this study, we examined the circadian rhythms of medical students and factors which may affect academic performance: perceived stress, sleep quality, time management. Students answered a Morningness-Eveningness questionnaire, the Time Management Personal Assessment questionnaire, the Pittsburgh Sleep Quality Index (PSQI), and the Perceived Stress Scale questionnaire. The correlation $(\mathrm{R}=0.87)$ between chronotype and GPA $(\mathrm{P}<0.001)$ showed a higher GPA in students with morning chronotypes. The PSQI analysis demonstrated $47 \%$ of the students had poor sleep quality; a correlation $(\mathrm{R}=-0.56)$ between the PSQI and GPA ( $\mathrm{p}<$ 0.04), supporting a link between higher GPA and good sleep. Results also showed a correlation $(\mathrm{R}=0.59)$ between time management and GPA ( $p<0.01)$, implying that the students with excellent time management skills $(17 \%)$ had excellent GPAs. Chronotypes, quality of sleep and time management, influence GPA and can be used as a guidance tool for academic advisors.
\end{abstract}

UDC Classification: 612/159.91; DOI: http://dx.doi.org/10.12955/cbup.v5.1056

Keywords: Circadian rhythms, time management organization, medical students

\section{Introduction}

Time management is one of the biggest problems of medical students. Good time management skills such as setting goals and monitoring the use of time can facilitate productivity and minimize stress, contributing to work effectiveness, maintaining balance and academic success (Al Khatib, 2014). Balduf (2009) recognized that poor time management skills can affect academic achievement negatively. Britton and Tesser (1991) found that the undergraduate students identified time management as their most pressing problem, which lead to anxiety, stress and poor sleep habits, and have a damaging effect on health and performance (Schneiderman et al., 2005). Not all time management methods work for everyone; (it depends on individual circadian rhythms (chronotypes). Understanding which circadian type is predominant in the body can help to assess what schedule is best. Laboratory studies which were conducted among medical students by Medeiros et al. (2001) showed that the irregularity of the sleep-wake cycle, the sleep deprivation, and internal desynchronization could be causing increased stress, and the stress could be influencing students' academic performances. Our body has daily rhythms of hormone concentrations, core temperature, and the sleep-wake cycle, all of which have wide spread effects on the function of the entire body (Saper et al., 2005). This rhythm is known as the circadian rhythm and is controlled by an internal body clock. Widely acknowledged individual differences in circadian rhythms, commonly called morningness and eveningness, indicate preferences associated with morning or evening activities. A morning chronotype is often awake between $6 \mathrm{AM}$ and $10 \mathrm{PM}$ and is most energetic in the morning. The evening chronotype is often awake between $9 \mathrm{AM}$ and $1 \mathrm{AM}$ and is most energetic in the afternoon and in the evening (Allebrandt et al., 2014). Studies conducted at the University of Zurich led by Steven Brown (Roenneberg, 2012) showed that the natural biological rhythms of 50\% of participants in the study were more or less out of step, and these participants often reported suffering from problems of insomnia or insufficient ability to concentrate on a task. Liaghatdar et al. (2016) also described the sleep-wake rhythm and internal rhythm of the body as having an effect on the cognitive activities of people and different people show different performances at different hours of the day. So everyone's body clock is not the same, therefore some difficulties synchronizing natural rhythms with daily plans can exist. Unfortunately, society is still organized to fit the early chronotypes for the people who prefer to work beginning early in the morning. Due to this mismatch between internal (circadian) and external (social) times, the people suffer from chronic sleep deficiency, which, in turn lead to stress, anxiety, and poor sleep habits (Van Dongen and Dinges, 2000). To solve this problem, following individual circadian rhythms and creating individual schedules is needed.

The present study was carried out to estimate the circadian rhythms of medical students and assess the correlation between their academic performance and chronotypes, sleep quality, and individual

\footnotetext{
${ }^{1}$ Trinity School of Medicine/ Saint Vincent \& the Grenadines, ymodna@trinityschoolofmedicine.org

2 Trinity School of Medicine/ Saint Vincent \& the Grenadines, bscott@ trinityschoolofmedicine.org
} 
subjective perception of stress. The purpose of the study was to use the results as a tool to help in the organization of student time management.

\section{Material and Methods}

The subjects were seventy-seven medical students of Trinity School of Medicine (TSOM) with mean age of 24.7 years $(\mathrm{SD}=2.3), 33$ male and 44 female. All students involved in this study had a similar academic load. Participation in the study was voluntary, and informed consent was obtained from all participants. Students who did not give consent and those who filled the questionnaire incompletely were excluded from this study. As variables, the chronotype, time management personal assessment, quality of sleep, and individual subjective perception of stress were analyzed. Student responses were linked to grade point average (GPA). Students were asked to answer the Morningness-Eveningness Questionnaire (MEQ) to determine their individual chronotypes. This MEQ (Horne and Ostberg (1976) consists of 19 items allow for the classification of objects into five types reflecting the student preference for morning or evening orientation. Scores for them are interpreted as follows: 16-30 extreme evening type; 31-41 - moderate evening type; 42-58 - indifferent type; 59-69 - moderate morning type; and 70-86 extreme morning type. Student time management was assessed via the scores of on Time Management Personal Assessment questionnaire (Britton and Tesser, 1991). This questionnaire includes 25 items which are scored by the following scale: 2 for "Always," 1 for "Sometimes," 0 for "Never." These data are interpreted as follows: 45-50 points - excellent time management skills; 30-44 points - time management is fairly well but should use the guide to increase the skills; 0-30 points - time management skills should be improved. The quality of sleep was assessed via their score on the Pittsburgh Sleep Quality Index (PSQI) questionnaire (Buysse et al., 1989), which consisted of 10 questions related to their normal sleep habits. Sleep quality was considered bad for individuals who obtained a score higher than 5. The Perceived Stress Scale (Cohen et al., 1983) was used for an evaluation of the level of stress. This scale includes 10 items and uses a 5 point rating scale ranging from 0 to 4: 0 - never; 1- almost never; 2 - sometimes; 3 - fairly often; 4 - very often; items 4 , 5,7 , and eight are reversed scored). Data obtained were interpreted regarding the following scale: 0-13 - low-stress level; 14-26 - moderate stress level; 27- 40 - high-stress level.

\section{Results and Discussion}

Chronotype distribution showed that the majority (55\%) of students had an indifferent type, $22.5 \%$ had a moderate morning type, $12.5 \%$ had a moderate evening and $10 \%$ had a definite morning chronotype (Figure 1). This distribution is most likely due to an attempt to synchronize natural rhythm with an academic schedule.

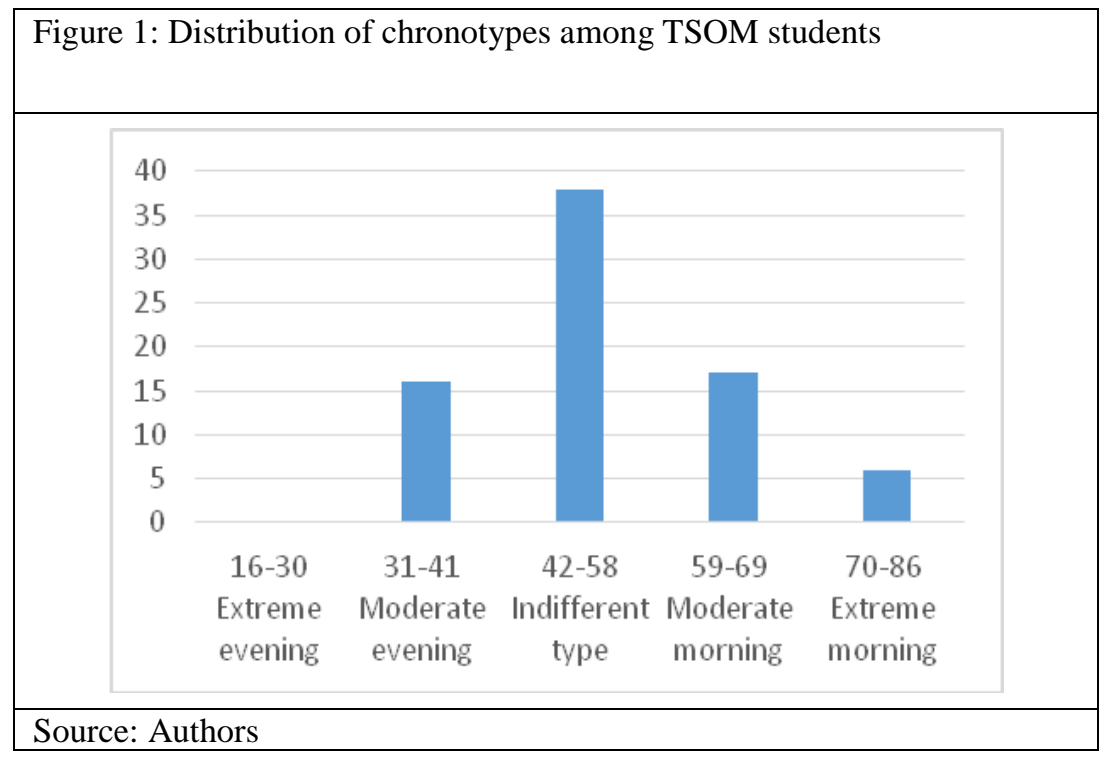

We also found the correlation $(\mathrm{R}=0.87)$ between chronotype and GPA which was significant $(\mathrm{P}<0.001)$, showed a higher GPA among students with morning chronotypes (Figure 2). 


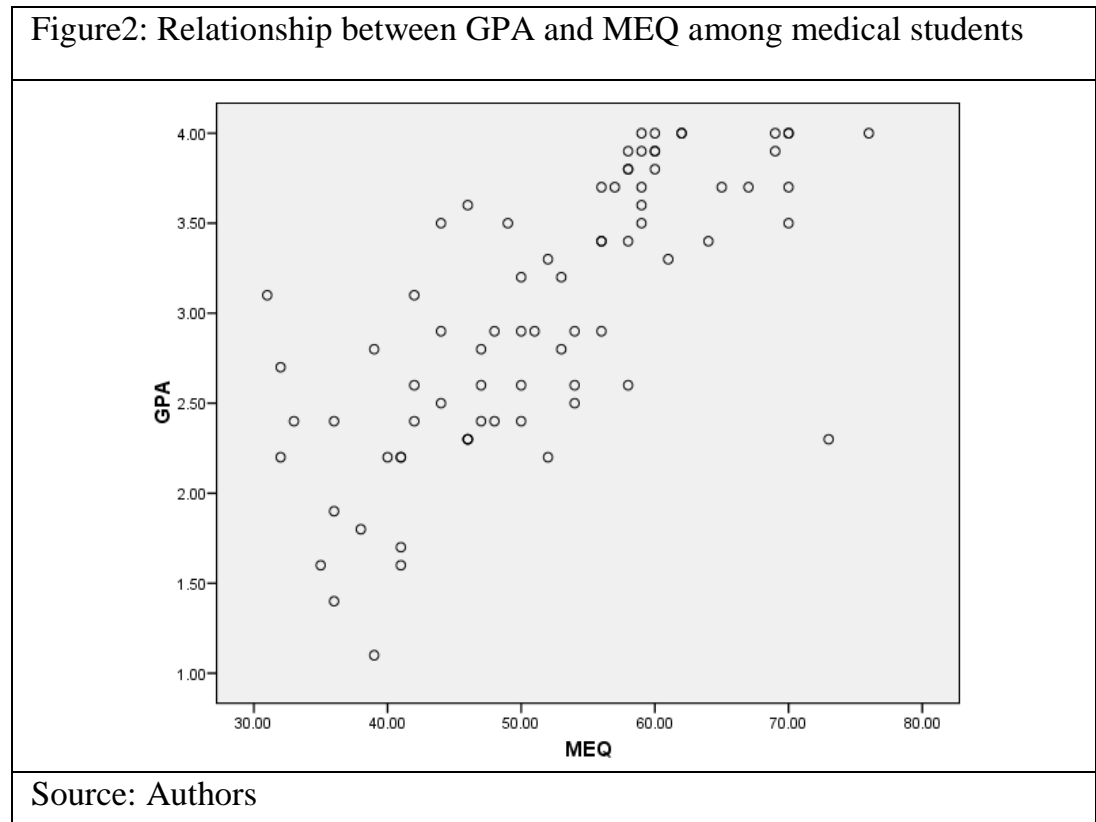

This indicated that the students with evening chronotype were maladapted for academic achievement whereas students with morning chronotype were adapted. One of explanation this finding may be "a synchrony effect in individuals" (May et al., 1998) suggesting morning-type individuals perform better at cognitive tasks in the morning and vice versa for evening-type individuals. This effect is based on cortisol levels which increased earlier and have higher amplitude in morning chronotypes than in evening chronotypes; therefore, morningness-oriented students reach their peak performances in the morning hours and eveningness-oriented students have a peak performances in the afternoon (Hasher et al., 2005). Liaghatdar et al. (2016) conducted research among medical students and concluded that evening students experienced academic failure in difficult courses, which required a more cognitive performance so there was a proposal to change the class hours of difficult specialized courses to the middle of the day. Enhancing the understanding and remembering abilities of morning and evening people were studied, and results of this study showed that immediate memory of morning people had a better performance in the early hours of the day and that of the evening people had a better performance in the evening hours (Song et al. (2000). In our research, we also studied the sleep quality of the TSOM students. The PSQI analysis showed $42 \%$ of the students had poor sleep quality. In addition, a correlation $(\mathrm{R}=-0.56)$ was found between the PSQI and GPA ( $\mathrm{p}<0.04)$, supporting an association between higher GPA and good sleep (Figure 3).

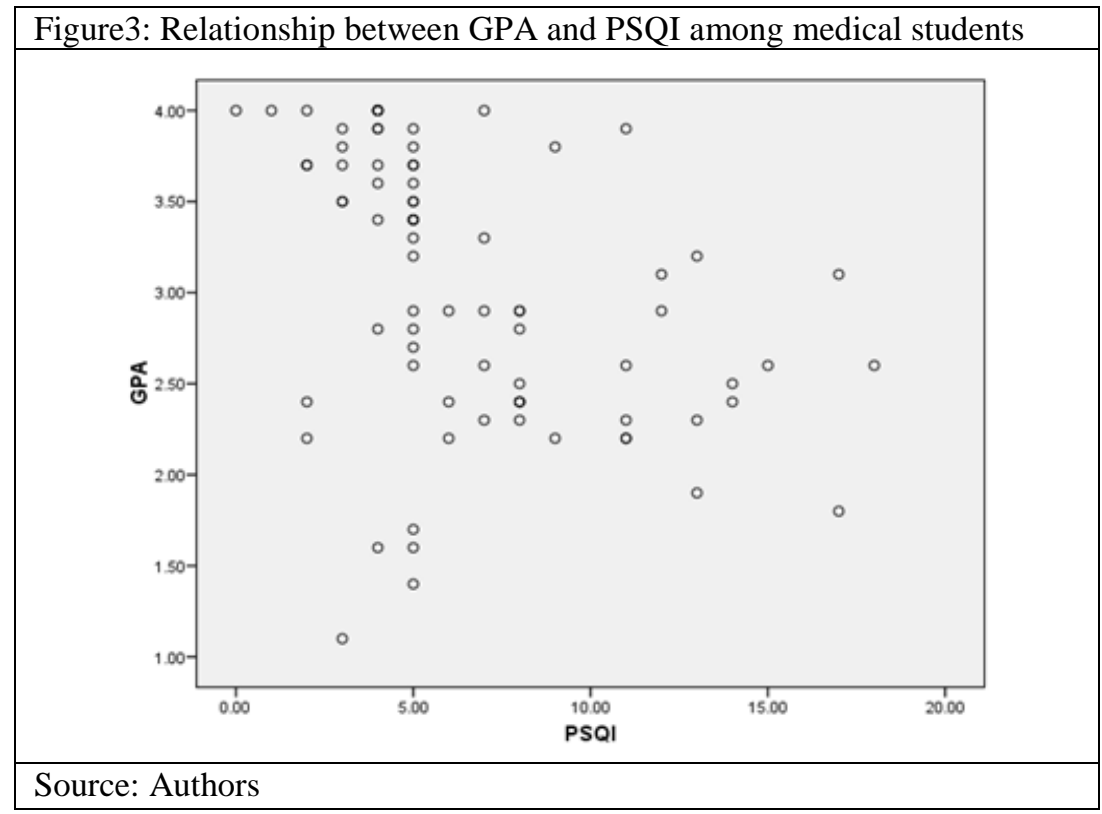


Hence, sleep deprivation affects eveningness-oriented students accompanied by academic difficulties. This might be explained by increased sleepiness and tiredness of evening students due to an early beginning their academic day, which creates some problems in adjusting to the schedule in medical school (Gau et al., 2004). There was no significant relationship between GPA and perceived stress level ( $p>0.7)$. This suggests a stress factor is a part of students' life. Stress in academic situations can have both positive and negative consequences. Misra and McKean (2000) suggested classifying stress as "unfavorable stress," which is associated with an inhibition of students' academic performance due to suppression of the learning process, and as "favorable stress," which creates a sense of competence and enhanced learning capability. We also found a positive correlation (Figure 4) $(\mathrm{R}=0.59)$ between the GPA and time management ( $\mathrm{p}<0.01)$, implying that the students with excellent time management skills (17\%) had excellent GPAs. Those with poor skills (14\%) had worse GPAs. However for 69\% of the volunteers, guidance is needed to increase skills, and as a result, it can increase the GPA. This explains why a majority of the university students complaining about running out of time when asked to do a certain task, they get frustrated because they are not able to complete the task before the deadline because they cannot manage their time. In turn this increases the level of 'unfavorable stress' which adversely impacts the GPA.

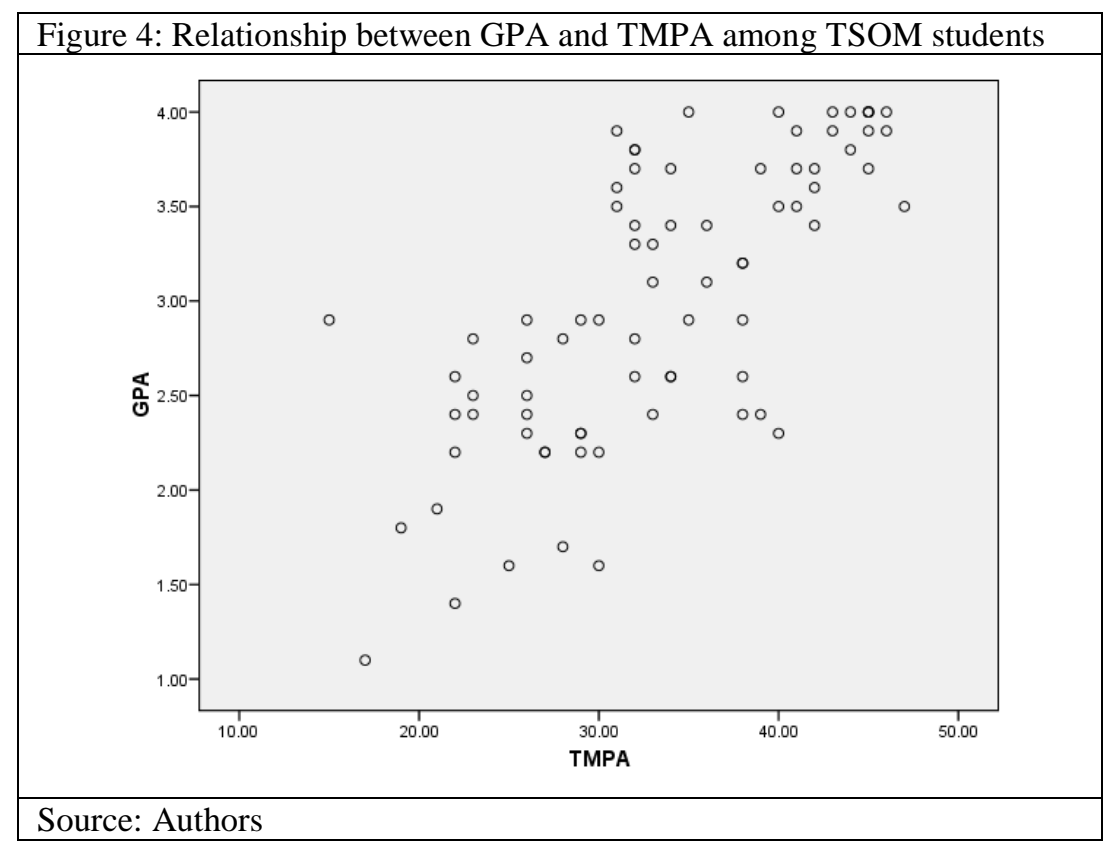

\section{Conclusion}

- Evening chronotype, poor quality of sleep, bad time management skills were significant negative predictors of the grade point average (GPA) among medical students.

- Therefore, for the improvement of GPA in medical school students should:

1. Consider personal circadian rhythms during the planning of study day and strive to improve their time management skills.

2. Understand the important role of sleep and naps during their academic life which provide short/long term recovery and increase the level of energy for a productive learning process. Sufficient hours for the day /and night sleep must be scheduled daily.

- In addition, medical universities should try to adopt the schedule of exams and learning activities to accommodate circadian rhythms of the students. The most difficult subjects and exams should be conducted between 10-12 am when the alertness is high for all circadian rhythms.

\section{References}

Al Khatib, A. Time Management and Its Relation to Students' Stress, Gender and Academic Achievement among Sample of Students at Al Ain University of Science and Technology, UAE. International Journal of Business and Social Research, 4 (5), 47-58. 
Allebrandt, K., TederLaving, M., Kantermann, T., Peters, A., Campbell, H., Wilson, J., Metspalu, A., Roenneberg, T. (2014). Chronotype and sleep

duration: the influence of season of assessment. Chronobiology International, 31(5), 731-740.

Balduf, M. (2009). Underachievement among college students. Journal of Advanced Academics, 20, 274-294.

Britton, B. \& Tesser, A. (1991). Effects of time management practices of college grades. Journal of Educational Psychology, 83, 405-410.

Buysse, D., Reynolds, C., Monk, T., Berman, S., Kupfer, D. (1989). The Pittsburg Sleep Quality Index: a new instrument for psychiatric practice and research. Psychiatry Research, 28, 193-213.

Cohen, S., Kamarck, T., Mermelstein, R. (1983). A global measure of perceived stress. Journal of Health and Social Behavior, 24, 385-396

Gau, S., Soong, W., Merikangas, K. (2004). Correlates of sleep-wake patterns among children and young adolescents in Taiwan. Sleep, 27 (3) 512-519.

Hasher, L., Goldstein, D., May, C. (2005). It's about time: Circadian rhythms, memory and aging. Human Learning and Memory: Advances in Theory and Application, 2, 199-218.

Horne, J. \& Ostberg, O. (1976). A self-assessment questionnaire to determine morningness-eveningness in human circadian rhythms. Internal Journal of Chronobiology, 4, 97-110.

Liaghatdar, M., Ashoorion, V., Avizhgan, M. (2016). Compare the educational achievement of medical students with different circadian thythms in difficult courses of basic sciences. Advanced Biomedical Research, http:// doi: 10.4103/22779175.178806

Management for health professions student. Retrieved from http://www.oshpd.ca.gov/HWDD/2011/HCTP/pdfs/TimeMgmtforHPStudents.pdf.

May, C. \& Hasher, L. Synchrony effects in inhibitory control over thought and action. (1998). Journal of Experimental Psychology: Human Perception \& Performance, 24(2) 363-379.

Medeiros, A., Mendes, D., Lima, P., John, F. (2001).

Relationships between Sleep-Wake Cycle and Academic

Performance in Medical Students. Biological Rhythm Research, 32 (2), 263-270

Misra, S. \& McKean, M. (2000). College student's academic stress and its relation to their anxiety, time management and leisure satisfaction. Retrieved from

https://www.researchgate.net/publication/209835950_College_students'academic_stress_and_its_relation_to_their_anxiety_ti me_management_and_leisure_satisfaction.

Roenneberg, T. (2012). Internal time. Retrieved from http://www.hup.harvard.edu/catalog.php?isbn=9780674065857\&content=bios

Saper, C., Cano G., Scammell, T. (2005) Homeostatic, circadian, and emotional regulation of sleep. Journal of Comparative Neurology, 493 (1), 92-98.

Schneiderman, N, Ironson, G, Scott, D. (2005). Stress and health: Phychological, Behavioral, Behavioral, and Biological Determinants, http:// doi: 10.1146/annurev.clinpsy.1.102803.144141

Song, J. \& Stough, C. (2000). The relationship between morningness-eveningness, time of day, speed of information processing, and intelligence. Personality and Individual differences, 29(1) 179-190.

Van Dongen, H. \& Dinges, D. (2000). Circadian rhythms in fatigue, alertness, and performance. Principles and Practice of Sleep Medicine, 20, 391-399. 